\title{
Cryptanalysing the Critical Group: Efficiently Solving Biggs's Discrete Logarithm Problem
}

\author{
Simon R. Blackburn \\ Department of Mathematics \\ Royal Holloway, University of London \\ Egham, Surrey TW20 0EX \\ United Kingdom \\ s.blackburn@rhul.ac.uk
}

November 7, 2008

\begin{abstract}
Biggs has recently proposed the critical group of a certain class of finite graphs as a platform group for cryptosystems relying on the difficulty of the discrete log problem. The paper uses techniques from the theory of Picard groups on finite graphs to show that the discrete log problem can be efficiently solved in Biggs's groups. Thus this class of groups is not suitable as a platform for discrete logarithm based cryptography.
\end{abstract}

Keywords: Critical group, discrete logarithm problem, cryptanalysis, chip-firing, Picard group.

AMS Classification: 94A60, 05C50

\section{Introduction}

Let $G$ be an abelian group written additively, and let $g, h \in G$ have the property that $h$ lies in the subgroup generated by $g$. The discrete logarithm problem asks for an integer $k$ such that $h=k g$. Groups where the discrete 
logarithm problem is hard are of great interest, since they can form the basis for key exchange protocols [4] and public key cryptosystems [5]. See Stinson [8] for a good introduction to the area. Of course, the difficulty of the discrete logarithm problem depends heavily on the representation of $G$. If the elements of $G$ are represented as integers in the range $[0,|G|-1]$ with addition in the group being integer addition modulo $|G|$, the discrete logarithm problem is efficiently solved by an application of the extended Euclidean algorithm. However, there are representations of $G$ in which the discrete logarithm problem is thought to be hard in general. (Examples include realisations of $G$ as a group of points on an elliptic curve, or as a subgroup of the multiplicative group of a finite field.)

Biggs [3] has recently proposed the critical group of a class of finite graphs as a candidate for a group with a hard discrete logarithm problem. The purpose of this paper is to show that, in fact, these groups have an efficiently solvable discrete logarithm problem and so are not suitable as platform groups for discrete logarithm based cryptography. (Here 'efficiently solvable' means two things: there is a polynomial time algorithm that solves the problem; there exists an algorithm that runs well for practical parameters.)

In the next section, we will recap Biggs's construction of his class of critical groups. In the final section of the paper we will show, using the theory of Picard groups on finite graphs, that the discrete logarithm problem can be efficiently solved.

\section{A chip-firing game on a graph}

The abelian group that Biggs proposes as a platform for the discrete logarithm problem is represented as a chip-firing game on a graph. See Biggs $[1,2]$ for background on this area. We define the group as follows.

Let $n$ be a positive integer (the security parameter of the problem). Let $W$ be the wheel graph with $2 n+1$ spokes. So $W$ consists of a cycle $v_{1}, v_{2}, \ldots, v_{2 n+1}$ (the 'rim' of the wheel) together with a 'hub' vertex $q$ that is joined to each of the vertices $v_{i}$. Let $W^{\dagger}$ be the graph obtained from $W$ by adding a vertex $v_{2 n+2}$ on the edge joining $v_{2 n+1}$ and $v_{1}$. We write $V$ for the set of vertices of $W^{\dagger}$ and $E$ for the set of edges of $W^{\dagger}$. We define $V^{*}=V \backslash\{q\}$, so $V^{*}$ is the set of vertices on the rim of the modified wheel graph.

A configuration on $W^{\dagger}$ (with distinguished vertex $q$ ) is a function $s: V \rightarrow$ $\mathbb{Z}$ such that $s(v) \geq 0$ for all $v \in V^{*}$ and such that $s(q)=-\sum_{v \in V^{*}} s(v)$. A 
firing of a vertex $u$ is a move from a configuration $s$ to a configuration $s^{\prime}$, where we reduce $s(u)$ by $\operatorname{deg} u$ and add 1 to each of the neighbours of $u$. So

$$
s^{\prime}(v)=\left\{\begin{array}{cl}
s(v)-\operatorname{deg} v & \text { if } v=u \\
s(v)+1 & \text { if } v \text { and } u \text { are adjacent } \\
s(v) & \text { otherwise. }
\end{array}\right.
$$

A firing of a vertex $u$ with $u \neq q$ is legal if $s(u) \geq \operatorname{deg} u$. A firing of $q$ is legal if there are no legal firings of a vertex $u$ with $u \neq q$. A configuration $s$ is stable if $q$ is the only vertex that can be fired legally. A stable configuration $s$ is critical if there is a non-empty sequence of legal firings that return to $s$. It is possible to show that given any starting configuration $s$, there is a unique critical configuration $\gamma(s)$ that can be reached by a sequence of legal firings starting at $s$.

The critical group $\mathcal{K}\left(W^{\dagger}\right)$ is the set of critical configurations, with addition of two configurations $s$ and $s^{\prime}$ defined to be $\gamma\left(s+s^{\prime}\right)$. Note that an element of $\mathcal{K}\left(W^{\dagger}\right)$ may be specified by the vector $\left(s(u)_{u \in V^{*}}\right)$ of length $2 n+2$. Since $0 \leq s(u) \leq \operatorname{deg} u \leq 3$ for all $u \in V^{*}$, this representation is efficient. Moreover, a result of van den Heuvel [6] shows that a group operation can be computed using at most $O\left(n^{3}\right)$ firings, and so group operations may be carried out fairly efficiently.

Let $f_{n}$ and $\ell_{n}$ be the $n$th Fibonacci and $n$th Lucas numbers respectively. Biggs [3] shows that $\mathcal{K}\left(W^{\dagger}\right)$ is cyclic of order $\tau$, where $\tau=2 \ell_{2 n+1} f_{2 n+2}$.

\section{A cryptanalysis using the Picard group}

This section shows that the discrete logarithm problem in $\mathcal{K}\left(W^{\dagger}\right)$ can be solved efficiently. We construct an isomorphism $\theta$ from $\mathcal{K}\left(W^{\dagger}\right)$ to the additive group $\mathbb{Z} / \tau \mathbb{Z}$ of integers modulo $\tau$. Constructing the isomorphism requires just $O(n)$ integer operations and the storage of $O(n)$ integers. Once this is done, an image under $\theta$ can be computed in $O(n)$ operations. Once $\theta$ has been constructed, we can solve the discrete logarithm problem as follows. Given $g, h \in \mathcal{K}\left(W^{\dagger}\right)$ with $h$ in the cyclic subgroup generated by $g$, we note that $\theta(h)$ lies in the subgroup generated by $\theta(g)$ and so we may find $k \in \mathbb{Z}$ such that $k \theta(g)=\theta(h)$ since the discrete logarithm problem is easy in $\mathbb{Z} / \tau \mathbb{Z}$. But then $k$ is a solution to our discrete logarithm problem in $\mathcal{K}\left(W^{\dagger}\right)$, and we are done. 
We construct $\theta$ as the composition of three isomorphisms $\phi, \psi$ and $\pi$, which we define below.

The Picard group $\operatorname{Pic}\left(W^{\dagger}\right)$ is an abelian group isomorphic to $\mathcal{K}\left(W^{\dagger}\right)$; see Biggs [1, Sections 28-32] for the relevant theory of this group. The Picard group can be thought of as a chip-firing game with a less restrictive definition of configuration and where all firings are legal. So an 'unrestricted' configuration is a labelling $s$ of the vertices of the graph by integers such that $s(q)=-\sum_{v \in V^{*}} s(v)$. We think of unrestricted configurations $s$ and $s^{\prime}$ as being equivalent if there is a sequence of firings that converts $s$ to $s^{\prime}$. The Picard group is the set of equivalence classes of unrestricted configurations under pointwise addition. Since $s(q)$ is determined by the labels of the vertices in $V^{*}$, we can think of the set of unrestricted configurations as a free abelian group of rank $\left|V^{*}\right|$. We add a relation to this free group for each firing at a vertex $u$. In fact, we need only add the $\left|V^{*}\right|$ relations corresponding to firings at vertices $u \neq q$ : the relation corresponding to $u=q$ is a consequence of the remaining relations.

A concrete realisation of $\operatorname{Pic}\left(W^{\dagger}\right)$ is as follows. Let $\mathbb{Z}^{2 n+2}$ be the free abelian group generated by $V^{*}$. Let $Q^{\prime}$ be the $(2 n+2) \times(2 n+2)$ matrix with rows and columns indexed by $V^{*}$ where

$$
Q_{v_{i} v_{j}}^{\prime}=\left\{\begin{array}{cl}
\operatorname{deg} v_{i} & \text { if } i=j \\
-1 & \text { if } i \neq j \text { and } v_{i} \text { and } v_{j} \text { are adjacent } \\
0 & \text { otherwise. }
\end{array}\right.
$$

Then the Picard group is the quotient of $\mathbb{Z}^{2 n+2}$ by the relations matrix $Q^{\prime}$. (Let $Q$ be the discrete Laplacian of $W^{\dagger}$. Then $Q^{\prime}$ is the result of removing the row and column indexed by the vertex $q$ from $Q$, and so by $[1$, Proposition 30.1] this construction is indeed a realisation of $\operatorname{Pic}\left(W^{\dagger}\right)$.)

Let $\phi: \mathcal{K}\left(W^{\dagger}\right) \rightarrow \operatorname{Pic}\left(W^{\dagger}\right)$ take a critical configuration $s$ to the obvious element of $\operatorname{Pic}\left(W^{\dagger}\right)$, namely the coset with representative $\left(s(u)_{u \in V^{*}}\right)$. Then $\phi$ is an isomorphism [1, Proposition 32.2]. It is clearly trivial to compute the image of an element under $\phi$.

Let $A$ be the Smith Normal Form of the relations matrix $Q^{\prime}$ above. So

$$
A=\operatorname{diag}(1,1, \ldots, 1,1, \tau),
$$

since $\mathcal{K}\left(W^{\dagger}\right)$ is cyclic of order $\tau$. Let $X, Y \in \mathrm{GL}(2 n+2, \mathbb{Z})$ be such that $X Q^{\prime} Y=A$. The matrices $X$ and $Y$ may be efficiently found by standard techniques using a polynomial number of integer operations. However, since 
$Q^{\prime}$ is of such a special form we can obtain the Smith Normal Form and the matrices $X$ and $Y$ much more efficiently than in general. To see this, first note that replacing $Q^{\prime}$ by $-Q^{\prime}$ and permuting its rows appropriately transforms $Q^{\prime}$ into the matrix $Q^{\prime \prime}$ where

$$
Q^{\prime \prime}=\left(\begin{array}{rrrrrrr}
1 & & & & & 1 & -2 \\
-3 & 1 & & & & & 1 \\
1 & -3 & 1 & & & & \\
& \ddots & \ddots & \ddots & & & \\
& & 1 & -3 & 1 & & \\
& & & 1 & -3 & 1 & \\
& & & & 1 & -3 & 1
\end{array}\right)
$$

But now it is easy to write down an explicit sequence of $O(n)$ elementary row operations that transforms $Q^{\prime \prime}$ into a matrix $R$ of the form

$$
R=\left(\begin{array}{cc}
I & M \\
0 & N
\end{array}\right)
$$

where $I$ is the $2 n \times 2 n$ identity matrix, 0 is the $2 \times 2 n$ all zero matrix and $M$ and $N$ are $2 n \times 2$ and $2 \times 2$ integer matrices respectively. The matrices $M$ and $N$ contain $O(n)$ entries and can be efficiently computed from the last two columns of $Q^{\prime \prime}$ by tracing the effect of the sequence of row operations on these columns. Finding matrices $X^{\prime}, Y^{\prime} \in \mathrm{GL}(2 n+2, \mathbb{Z})$ such that $X^{\prime} R Y^{\prime}$ is in Smith Normal Form now takes just $O(n)$ operations (using standard algorithms), and since $R$ is obtained from $Q^{\prime}$ using row operations alone we find that we may take $Y=Y^{\prime}$. Note that $Y$ is a sparse matrix, containing just $O(n)$ non-zero entries. Also note that we may work modulo $\tau$ to avoid problems with coefficients becoming too large: see for example Sims [7, Section 8.4].

Let $G$ be the quotient of $\mathbb{Z}^{2 n+2}$ by the relations matrix $A$. Define $\psi$ : $\operatorname{Pic}\left(W^{\dagger}\right) \rightarrow G$ to be the map induced by right multiplication by $Y$. Then (see [7, Proposition 8.3.3]) $\psi$ is an isomorphism. Clearly $\psi$ may be computed using $O(n)$ integer operations.

Finally, let $\pi: G \rightarrow \mathbb{Z} / \tau \mathbb{Z}$ be the map induced by projecting onto the last co-ordinate; once more it is clear that $\pi$ is easy to compute.

If we define $\theta=\pi \psi \phi$, we have found an isomorphism with the properties we need; so the discrete logarithm problem is efficiently solvable in $\mathcal{K}\left(W^{\dagger}\right)$ as claimed. 
Acknowledgement The author would like to thank Steven Galbraith for his useful comments on an earlier draft of this paper.

\section{References}

[1] N.L. Biggs, 'Algebraic potential theory on graphs', Bull. London Math. Soc. 29 (1997) 641-682.

[2] N.L. Biggs, 'Chip-firing and the critical group of a graph', J. Algebraic Combin. 9 (1999) 25-45.

[3] N.L. Biggs, 'The critical group from a cryptographic perspective', Bull. London Math. Soc. 39 (2007) 829-836.

[4] W. Diffie and M.E. Hellman, 'New directions in cryptography', IEEE Trans. Inform. Theory 22 (1976), 644-654.

[5] T. ElGamal, 'A public key cryptosystem and a signature scheme based on discrete logarithms', IEEE Trans. Inform. Theory 31 (1985), 469-472.

[6] J. van den Heuvel, 'Algorithmic aspects of a chip-firing game', Combin. Probab. Comput. 10 (2001) 505-529.

[7] Charles C. Sims, Computation with Finitely Presented Groups, Cambridge University Press, UK, 1994.

[8] Douglas R. Stinson, Cryptography: Theory and Practice (2nd Edition) Chapman and Hall/CRC Press, Boca Raton, 2002. 